there is a thermodynamic preference for it to bind to $S$ rather than to $R$; and when $\mathrm{T}$ is in its excited state, kinetic factors greatly favour energy transfer to $S$ compared with transfer to $R$. Impressively, the authors demonstrated that they could even use their method to convert a sample of the $S$-isomer of the tert-butylbearing allene (which had an e.e. of 95\%) to the $R$-isomer (which had an e.e. of $96 \%$ ).

Hölzl-Hobmeier and colleagues did not make a wide survey of which chemical groups could be attached to the allenes without disrupting the enantioselectivity of the deracemization, so this remains to be explored. However, groups that could disturb the hydrogen bonding between $\mathrm{T}$ and the allene would need to be avoided or protected (temporarily converted into another group that does not interfere with the hydrogen bonding). Nevertheless, the authors show that 17 racemic allenes bearing a variety of groups (see Fig. 3 of the paper ${ }^{1}$ ) can be deracemized to produce single enantiomers of $89-97 \%$ e.e. in good to excellent chemical yields (52-100\%). These e.e. values far exceed the value (3.4\%) obtained for the first reported deracemization of an allene ${ }^{6}$ in the early days of photochirogenesis research. Another attractive feature of the new method is that it uses a small amount of catalyst (only $2.5 \mathrm{~mol} \%$ compared with the amount of allene used).

The authors' findings unequivocally demonstrate that supramolecular photochirogenesis, when appropriately designed, can be a powerful tool for asymmetric synthesis that cannot be achieved using conventional, heatactivated reactions. The new reactions might be limited by the need to append hydrogenbonding groups to both the substrate and the photosensitizing template, and by the narrow range of compounds to which they are immediately applicable (which include sulfoxides and binaphthyl compounds). Nevertheless, the general concept and methodology, as well as the mechanistic details revealed by this study, will generate much discussion and open up fresh avenues of research.

Cheng Yang is in the Key Laboratory of Green Chemistry \& Technology of Ministry of Education, College of Chemistry, State Key Laboratory of Biotherapy, and Healthy Food Evaluation Research Center, Sichuan University, Chengdu 610064, China.

Yoshihisa Inoue is in the Department of Applied Chemistry, Osaka University, Suita 565-0861, Japan.

e-mail:inoue@chem.eng.osaka-u.ac.jp

1. Hölzl-Hobmeier, A. et al. Nature $\mathbf{5 6 4 , 2 4 0 - 2 4 3}$ (2018).

2. Yang, C. \& Inoue, Y. Chem. Soc. Rev. $\mathbf{4 3}, 4123-4143$ (2014).

3. Rodriguez, O. \& Morrison, H. J. Chem. Soc. D Chem. Commun. 679 (1971)

4. Alonso, R. \& Bach, T. Angew. Chem. Int. Edn $\mathbf{5 3}$, 4368-4371 (2014).

5. Ley, C., Morlet-Savary, F., Jacques, P. \& Fouassier, J. P. Chem. Phys. 255, 335-346 (2000).

6. Drucker, C. S., Toscano, V. G. \& Weiss, R. G. J. Am. Chem. Soc. 95, 6482-6484 (1973).

MICROBIOLOGY

\title{
Bacterial molecules target viral DNA
}

Bacteria can use specific protein-based strategies to defend individual cells against viruses. Evidence that bacterial small molecules also target viruses provides fresh insights into how bacteria thwart viral infection. SEE LETTER P.283

\section{MARTHA R. J. CLOKIE}

$\mathrm{T}$ o enjoy beautiful environments, we might need to defend ourselves against the resident pests, from midge flies on Scottish hillsides to mosquitoes in tropical jungles. If pests are numerous and diverse, a broad-spectrum defence strategy, such as spraying an insect repellent, can be best. Bacteria can also use general defences to combat their viral predators, in addition to having a plethora of more-specific defences that target particular viruses. On page 283, Kronheim et al. ${ }^{1}$ report their analysis of an antiviral defence system that can protect more than one bacterial species. These findings could have major implications for our understanding of how bacteria and viruses interact.

Viruses that infect bacteria are known as bacteriophages, or just phages, and they have key roles in shaping bacterial evolution, population dynamics and physiology. Phages are considered to be the most abundant and diverse biological entities on Earth ${ }^{2}$, and it is essential to consider them when trying to gain a full understanding of the bacterial world. Yet despite their importance, there are huge gaps in our knowledge. In many cases, information about phage host ranges (the types of bacterium that a particular phage can infect) is limited. Certain aspects of how bacteria defend themselves against phage attack are also mysterious.

Most bacterial species make numerous and diverse metabolites (small-molecule products of metabolism) that can provide widespread protection against attack from fungi and other types of bacterium. By contrast, most of the well-understood anti-phage defences in bacteria involve proteins, which often offer protection only at the level of the individual cell that makes the protein, rather than providing protection for a bacterial population. One such common bacterial defence is modification of the microbial cell surface to prevent phage attachment. Another strategy, called the CRISPR-Cas defence system ${ }^{3}$, depends on an infected bacterium recognizing and capturing sequences from the viral genome and using these to prime a response that kills viruses containing a copy of the captured sequences. Some bacteria take the approach of adding methyl groups to their DNA and degrading all unmethylated, and therefore foreign, $\mathrm{DNA}^{4}$. Many other fascinating examples of these 'single-cell' defence strategies exist ${ }^{5}$.

Broad-spectrum antiviral defence mechanisms in bacteria do occur but are less well known. For example, bacteria can shed vesicles from their outer membranes to 'mop up' phages ${ }^{6}$. The shortfall of examples in this category probably reflects the limited scope of previous research rather than a lack of such systems per se. Bacteria and phages have coevolved over approximately 3.9 billion years ${ }^{7}$, so it seems reasonable to speculate that nonspecific mechanisms might have a key role in bacterial defences. Arguably, such broad-based systems might have a longer evolutionary history than do the more-specific types of defence, and might have shaped the development of the subsequent targeted strategies.

Kronheim and colleagues began to investigate how bacteria might target phages by testing the ability of a total of 4,960 molecules from

"One type of
molecule can
defend diverse
species of
bacterium
against many
different types
of phage."
a drug-discovery library to prevent a phage called lambda from infecting the model bacterium Escherichia coli. This revealed 11 molecules that can limit the success of phage infection. Nine of these can embed within DNA and are called DNA-intercalating agents. Out of the 11 molecules, 4 belong to a group known as the anthracyclines. These include the naturally occurring compounds daunorubicin and doxorubicin, which are used as anticancer drugs. The dual ability of these molecules to target cancer cells and phages raises the question of whether they act by recognizing modified DNA.

The anti-phage effects of daunorubicin and doxorubicin were first discovered more than 50 years ago ${ }^{8-10}$. Yet, strangely, insights ${ }^{8-10}$ that bacteria can produce DNA-intercalating agents that target phages did not come to prominence. Research ${ }^{11-14}$ from the 1940s and 1950s also demonstrated that several other antibiotics could prevent phage infection. However, these observations were not interpreted as an indication that the molecules were 

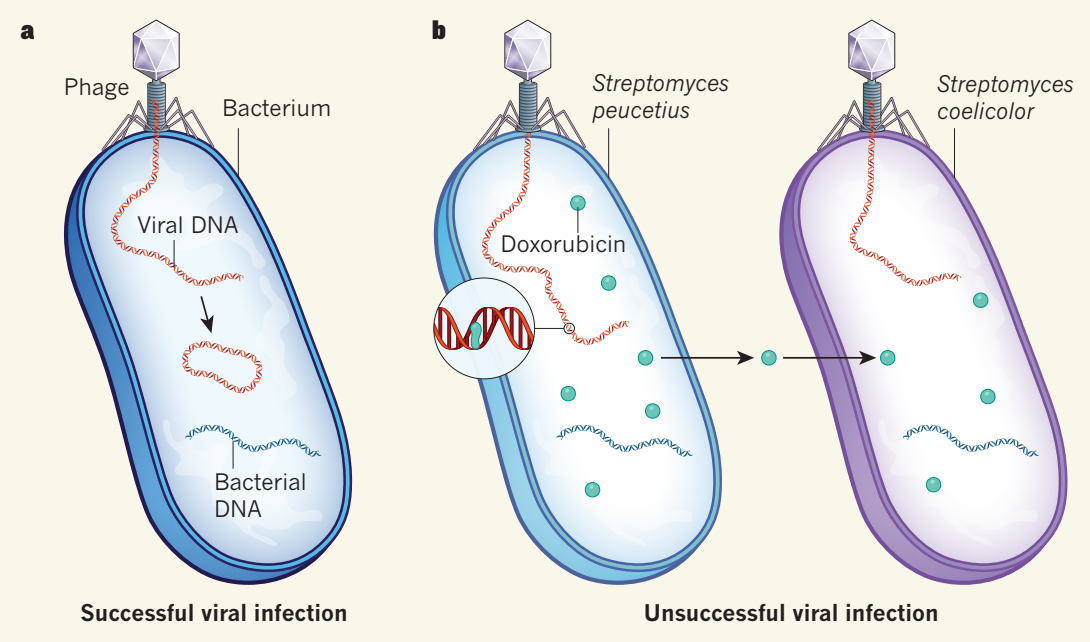

Figure 1 | A bacterial defence approach uses molecules to target viral DNA. a, During an early step in the infection of a bacterium by viruses called phages, the linear viral DNA (red) becomes circularized. b, Kronheim et al. ${ }^{1}$ report that bacteria from the genus Streptomyces make molecules that can block a successful viral infection. The molecules they identified with this property, such as doxorubicin, are DNA-intercalating compounds - they can become embedded in DNA. These intercalating molecules seem to affect viral rather than bacterial DNA. The authors' results suggest that these molecules block an early stage of viral infection, which might be the step at which viral DNA becomes circularized. Kronheim and colleagues report experimental results consistent with a model in which doxorubicin present in the medium of a culture of Streptomyces peucetius bacteria can enter Streptomyces coelicolor bacteria and protect them from phage infection. This reveals a broad-spectrum defence mechanism that could offer antiviral protection for bacterial populations from multiple species.

components of a natural bacterial anti-phage defence strategy.

Kronheim et al. sought to establish how the molecules they identified act to block phage infection. They demonstrated that viral entry into the cell, viral DNA replication, viral protein synthesis and virus assembly are not inhibited by the addition of daunorubicin. However, they found that daunorubicin can block a step immediately after viral entry and before replication. There will undoubtedly be future studies to determine the mechanism of molecular action at this stage. The most plausible hypothesis suggested by the authors is that daunorubicin blocks the circularization of linear viral DNA. If so, viral DNA that remains in a linear form might be degraded by the host bacterium, or phage infection might be suppressed because the viral DNA cannot interact with the proteins needed for its transcription.

Bacteria from the genus Streptomyces are particularly prolific metabolite producers and the source of numerous antibiotics. Kronheim and colleagues provide a crucial demonstration that Streptomyces species can produce daunorubicin and doxorubicin, revealing that bacteria can make their own metabolite-based anti-phage system. The authors showed that Streptomyces produce many anthracycline-like compounds, some of which prevent the infection of bacteria by specific phages, whereas others prevent infection by a range of phages. The authors tested samples of small-molecule extracts from Streptomyces species, and found that $30 \%$ of the extracts inhibited phage infection but did not affect bacterial growth, suggesting that bacterial DNA is not susceptible to interference by the molecules that hinder phage infection.

The authors' results suggest that Streptomyces bacteria release anthracyclines that can diffuse out of the bacterial cell into the external environment, enter neighbouring bacterial cells, and inhibit phage infection. This was confirmed by adding the medium from several-day-old cultures of Streptomyces to fresh cultures of Streptomyces to which phages were added. Remarkably, they showed that when doxorubicin-containing, microbefree medium from cultures of Streptomyces peucetius bacteria was added to cultures of Streptomyces coelicolor bacteria, it protected S. coelicolor from phage infection (Fig. 1).

The defence mechanism uncovered by Kronheim and colleagues contrasts with most known bacterial antiviral mechanisms in two ways. First, it uses metabolites rather than proteins. Second, the mechanism protects not just the cell that produces the anti-phage molecule, but also neighbouring bacterial cells of the same, and even different, bacterial species. This suggests a broad-spectrum metabolitebased defence system that acts in a manner akin to a 'phagicide', whereby one type of molecule can defend diverse species of bacterium against many different types of phage.

By expanding the concept of phage defence from the individual cell to the masses, Kronheim and colleagues' study suggests that phage defence is not as targeted to particular phages as was previously thought. Their work raises many questions. How important is this mechanism, and how are these metabolites made? Are they continually produced, or made only in response to phage infection? It would be interesting to learn how many different types of metabolite are able to target phages, how specific the metabolites' modes of action are, and to what extent such molecules can provide protection across different bacterial species.

Phages have developed ways to overcome most bacterial defences, and can cooperate to evade CRISPR-Cas defences ${ }^{15,16}$, so it seems probable that some phages might have developed ways to combat these bacterial defence molecules. Investigating whether this is the case should provide some interesting insights. The concept of phagicides is likely to spark searches for other types of anti-phage metabolite, perhaps leading to the discovery of antiviral metabolites that target other sorts of phage, such as those that have their genetic information in the form of RNA rather than DNA.

Kronheim and colleagues' work also adds to the growing body of evidence revealing the complexity of interactions between phages and bacteria. It follows other paradigm-shifting observations in this research area, such as the report that signalling between phages can affect whether the viruses enter a dormant state or replicate ${ }^{17}$. Building on Kronheim and colleagues' work, it is now time to consider the idea that metabolites can move from bacterium to bacterium to block phage infection. As additional systems are studied, this should help to unravel the extent of this communication, and illuminate how bacteria and their viral predators shape the world in which we live.

Martha R. J. Clokie is in the Department of Genetics and Genome Biology, University of Leicester, Leicester LE1 7RH, UK.

e-mail:mrjc1@le.ac.uk

1. Kronheim, S. et al. Nature 564, 283-286 (2018).

2. Clokie, M. R., Millard, A. D., Letarov, A. V. \& Heaphy, S. Bacteriophage 1, 31-45 (2011).

3. Makarova, K. S. et al. Nature Rev. Microbiol. 12, 722-736 (2015).

4. Labrie, S. J., Samson, J. E. \& Moineau, S. Nature Rev. Microbiol. 8, 317-327 (2010).

5. Doron, S. et al. Science 359, eaar4120 (2108).

6. Manning, A. J. \& Kuehn, M. J. BMC Microbiol. 1, 258 (2011).

7. Bamford, D. H. Res. Microbiol. 154, 231-236 (2003).

8. Sanfilippo, A. \& Mazzoleni, R. Giorn. Microbiol. 12, 83-92 (1964).

9. Parisi, B. \& Soller, A. Giorn. Microbiol. 12, 183-194 (1964).

10. Morita, J., Tanaka, A., Komano, T. \& Oki, T. Agric. Biol. Chem. 43, 2629-2631 (1979).

11.Weiss, U., Strelitz, F., Flon, H. \& Asheshov, I. N. Arch. Biochem. Biophys. 74, 150-157 (1958).

12.Strelitz, F., Flon, H., Weiss, U. \& Asheshov, I. N. J. Bacteriol. 72, 90-94 (1956).

13.Strelitz, F., Flon, H. \& Asheshov, I. N. J. Bacteriol. 69 280-283 (1955).

14.Asheshov, I. N., Strelitz, F., Hall, E. \& Flon, H. Antibiot. Chemother. (Northfield, III.) 4, 380-394 (1954).

15.Landsberger, M. et al. Cell 174, 908-916 (2018).

16.Borges, A. L. et al. Cell 174, 917-925 (2018).

17.Erez, A. et al. Nature 541, 488-493 (2017).

This article was published online on 5 December 2018. 